ARTICLE

\title{
Revisiting a basic question: does growing up in either female or male environment affect sex differences in academic strengths and occupational choices?
}

Daniel Fellman ${ }^{1 凶}$, Richard Bränström ${ }^{1} \&$ Agneta Herlitz ${ }^{1 凶}$

Are we affected by growing up in either female or male environment? This study examined whether girls' and boys' academic strengths at age 16 in verbal/language school subjects, relative to technical/numerical subjects, and cognitive demands of a chosen occupation at age 35 are influenced by having same- or opposite-sex siblings. Using representative population data from Swedish registers, we extracted (Study 1) 3-sibling families $(N=17,233)$, focusing on the mid-born, and (Study 2) 2-sibling families $(N=118,688)$, focusing on the last-born child. Both studies demonstrated that individuals' academic strengths were unaffected by sibship composition. Study 2 showed that boys with a sister tended to choose more numerically demanding occupations as compared to boys with a brother. Taken together, growing up in a more or less female or male environment, that is, having same- or opposite-sex siblings does not impact one's tendency to be academically more or less verbally or numerically aligned.

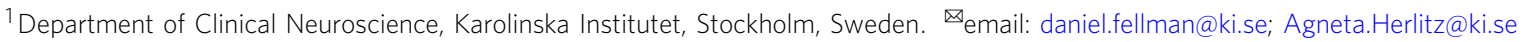




\section{Introduction}

re we affected by growing up in a more or less female or male environment? Does having individuals of the same sex around us reinforce or reduce sex differences and sextypicality in our cognitive abilities and cognitive demands of a chosen occupation? Social learning theories would posit that the acquisition of sex-role behaviour is dependent on children modelling themselves on same-sex individuals (Brim, 1958; Koch, 1955; McHale et al., 1999). If correct, having opposite- instead of same-sex siblings may affect behaviours that demonstrate sex differences. Here, we investigate whether individuals at 16 years of age born in same-sex sibship compositions differ in their relative academic strengths in comparison to individuals born in opposite-sex sibship compositions. In addition, we probe for potential long-lasting effects of sibship composition by examining whether these groups differ with respect to cognitive demands in their chosen occupation at age 35 .

A large literature on sex differences in academic strengths demonstrates that females often perform better than males in tasks assessing verbal fluency, verbal production, reading comprehension, and verbal episodic memory (Asperholm et al., 2019; Hyde and Linn, 1988; Maylor et al., 2007; Stoet and Geary, 2013). Males, on the other hand, tend to perform at a higher level on spatial tasks (Voyer et al., 1995, 2017), and on some numerical tasks (Lindberg et al., 2010; Stoet and Geary, 2018; Weber et al., 2014). Not surprisingly, these sex differences are mirrored in academic performance-although the female advantage in grade point average (GPA) extends to most school subjects, the female advantage tends to be larger in language subjects and smaller in mathematics and technical subjects (Dekhtyar et al., 2018; Voyer and Voyer, 2014).

Another way of illustrating sex differences in academic abilities is to assess individuals' relative intraindividual academic strengths. Research shows that more girls than boys have a relative strength in verbal/language school subjects over technical/numerical ones, whereas more boys than girls have a relative strength in technical/ numerical school subjects over verbal/language ones (Dekhtyar et al., 2018). The same pattern is found when contrasting males' and females' relative performance on verbal vs. spatial tasks (Stoet and Geary, 2018; Strandqvist et al., 2019).

It is well-known that the distribution of men and women is uneven across career paths, with women being underrepresented in, for instance, engineering and overrepresented in the humanities and social sciences (Ceci et al., 2014; Statistics Sweden, 2020). Research also indicates that individuals pursue occupations matching their academic strengths, contributing to more females than males selecting verbally demanding occupations, and more males than females selecting numerically demanding occupations (Dekhtyar et al., 2018).

The environment in which individuals are raised may reinforce or reduce sex differences in behaviours demonstrating sex differences. One potentially important environmental factor is the sex of any siblings, as having same- or opposite-sex siblings will lead to, for instance, more or less exposure to same- or oppositesex gender-typed play-behaviour (Todd et al., 2018). It has also been proposed that sex-typical behaviour is elicited by sibling competition for parents' limited resources and attention (Hamilton, 1967), that siblings affect each other by learning sextypical and sex-typical behaviours from one another (Detlefsen et al., 2018; McHale et al., 1999), and that sex-typical behaviour is elicited as a result of gender-differentiating parenting rather than how siblings interact with each other (Feinberg et al., 2003). Here, we investigate whether growing up in either female or male sibling environment affects future abilities and choices that typically demonstrate sex differences.

The pioneering studies focusing on children in toddlerhood (Brim, 1958; Koch, 1955) reported more gender-typical behaviour (e.g., gender-typical interests and play behaviour) (Su et al., 2009; Todd et al., 2018) in the presence of a same-sex sibling, such that children with a same-sex sibling exhibited more traditionally gender-typical behaviour than those with a sibling of the opposite sex. However, results from subsequent studies examining the relationship between sibship compositions and gender-typical behaviour have been mixed (Golsteyn and Magnée, 2020; Grotevant, 1978; Peter et al., 2018). Critically, research specifically examining whether early sibship composition influences one's cognitive strengths and career choices is lacking. Of the few existing studies examining educational and occupational choices, Gabay-Egozi et al. (2020) showed that, regardless of an individual's sex, the likelihood of preferring a STEM field (science, technology, engineering, math) increased with a greater proportion of boys in a sibship composition. Moreover, among secondborn boys, the likelihood of pursuing a STEM college major increased with $12 \%$ if an older sibling was male rather than female. In contrast, Brenøe (2018) found that girls with a brother tend to choose more female-dominated occupations relative to girls with a sister.

We conducted two studies utilizing high-quality register data on the full Swedish cohorts born 1973-1982, using normalized school grades from 9th grade (age 16; the last year of compulsory school), together with a follow-up where we examined the cognitive demands of their chosen occupation $\sim 20$ years later. Study 1 focused on the mid-born child in 3-sibling families, who could have either same-sex or opposite-sex siblings, and Study 2 focused solely on the last-born child in 2-sibling families with either a same-sex or opposite-sex older sibling. In both studies, we examined the impact of sibship composition on two target outcomes known to show sex differences: (1) academic strengths at age 16 and (2) cognitive demands of the chosen occupation at age 35. Moreover, we (3) examined whether the association between cognitive demands of chosen occupation at age 35 and sibship composition was moderated by the individuals' academic strengths at age 16 . If our tendency to be more or less verbally or numerically aligned is influenced by growing up in a more or less female or male environment, we should expect girls and boys with same- or opposite-sex siblings to differ in regard to their academic strengths and in the cognitive demands of their chosen occupations.

\section{Methods}

Study 1 focused solely on the mid-born child in 3-sibling families. Specifically, we investigated whether a mid-born girl with an older and younger sister (GGG) would show different academic strengths and make different occupational choices relative to mid-born girls with an older and younger brother (BGB). Similarly, we examined whether a mid-born boy with a younger and older brother (BBB) would exhibit different academic strengths and occupational choices relative to mid-born boys with a younger and older sister (GBG). In Study 2, we focused exclusively on the last-born child in 2-sibling families. Here, we examined whether last-born girls with an older sister (GG) would show different academic strengths and make different occupational choices relative to last-born girls with an older brother (BG). Likewise, we examined whether last-born boys with an older brother (BB) would exhibit different academic strengths and occupational choices relative to last-born boys with an older sister (GB).

Participants and procedure. Information from several nationwide Swedish population registers (e.g., Multi-generation register; National School Register; Swedish Register of Education; Swedish 
Occupational register) were used to retrieve longitudinal information on the socio-economic, demographic, educational, and occupational situation for the entire Swedish population born between 1973 and 1982 ( 800,000 individuals).

Study 1. Data preprocessing began with retrieving all 3-sibling families in Sweden to whom the mid-born child was born between 1973 and 1982. For children born during this time period in Sweden, scores on standardized, subject-specific national tests largely directed a student's school grades. As these national tests were normalized for a national average of 3.0 (of 5.0) and a standard deviation of 1, the resulting school grades-available in the Swedish population registries-provide detailed information about specific school subjects that is comparable across the investigated cohorts. Altogether, we identified 111,744 3-sibling families where the mid-born child was born within the aforementioned time intervals. Of those, 56,801 midborn children grew up in family composition that did not meet our predefined criteria (excluded compositions: BBG, BGG, GBB, GGB), leaving a sample size of 54,943 mid-born children. To increase the likelihood that the mid-born child had grown up with sufficient exposure to his/her siblings, only those mid-born children whose younger sibling was between 1-5 years younger and whose older sibling was between 1 to 5 years older were included. This resulted in the exclusion of 29,297 mid-born children, and left a sample size of 25,646 children. Of these children, 4396 who had not completed 9th grade between the years 1988 and 1997 (the time period available in the registries during which Sweden applied the normalized grading system) were excluded, leaving 21,250 children. For these remaining children $(n=2359)$, the four 9 th grade school subjects relevant for calculating the academic strengths score were not available, resulting in a further reduction to 18,891 mid-born children. Furthermore, 1567 children were excluded for having missing data in one or more of the following variables: education, occupation, birth country, mother's education, mother's biological age at birth. These exclusions resulted in a final sample size of 17,233 children. Of those, 8349 (48.4\%) were females (GGG $n=4317$; BGB $n=4032)$, and $8884(48.4 \%)$ were males (BBB $n=5205$; GBG $n=3679$ ).

Study 2. As in Study 1, data preprocessing began by extracting all 2 -sibling families in Sweden to whom the last-born child was born between 1973 and 1982. We identified 222,107 2-sibling families where the last-born child was born within the aforementioned time intervals. Of these, 49,792 last-born children had an older sibling who was either $<1$ or $>5$ older than him/her and was excluded, leaving 172,315 children. Of these children, 27,550 had not completed their 9th grade between the years 1988 and 1997, leaving a sample size of 144,765 last-born children. The four 9th grade school subjects relevant for calculating the academic strengths score were not available for 15,239 children, resulting in a further reduction to 129,526 last-born children. Furthermore, 26,077 children were excluded for having missing data in one or more of the following variables: education, occupation, birth country, mother's education, mother's biological age at birth. These exclusions resulted in a final sample size of 117,915 children. Of those, 58,105 (49.3\%) were females (GG $n=26,528$; BG $n=31,577)$, and 59,810 (50.7\%) were males (BB $n=28,794$; GB $n=31,016)$.

\section{Materials}

Academic strengths at age 16. The participants' academic strengths were retrieved and calculated from the National School Register. From 1988 onwards, this register includes information on each Swedish child's educational achievements in individual school subjects. We used school data collected from 1988 to 1997, the time period during which the school system employed a normally distributed scale of five grades spanning from 1 to 5 , where 1 was the lowest and 5 the highest.

We calculated an academic strengths score based on the work conducted by Dekhtyar and colleagues (2018) using grades from four school subjects, namely Swedish, English, Math and Technics. Technics in the Swedish school covers a wide range of sub-subjects related to technical systems, energy, and technological change. The verbal subjects, Swedish and English, were selected to tap into participants' verbal/language performance, whereas Technics and Math were selected to tap into participants' technical/numerical performance. The decision to use these four subjects was based on research showing reliable sex differences favouring females in verbal tasks (Asperholm et al., 2019; Hyde and Linn, 1988; Maylor et al., 2007; Stoet and Geary, 2013) and favoring males in spatial and numerical tasks (Voyer et al., 1995, 2017).

The calculation procedure for the academic strengths scores began with separate summations of verbal subjects scores (Swedish and English; potential range 2-10) and numerical subjects scores (Technics and Math; potential range 2-10), after which the summed verbal scores were subtracted from the summed numerical scores. Next, as only very few had an academic strengths score $<-4$ (Study $1 n=13$; Study $2 n=67$ ) and $>4$ (Study $1 n=8$; Study $2 n=52$ ), we imputed these extreme values to -4 and 4 , respectively. For each participant, a negative academic strengths score indicates that the summed grades assigned in verbal/language subjects (Swedish and English) were lower than the summed grades obtained in numerical/technical subjects (Technics and Math), indicating a relative academic strength in numerical/technical subjects. A positive academic strengths score would indicate the opposite.

Cognitive demands of chosen occupation at age 35 . The participants' occupational information was retrieved from the longitudinal database STATIV (Statistics Sweden, Documentation of STATIV 2018). The occupational information was collected from the year the participant turned 35 years, which fell in the time period 2008-2017 for the individuals in our cohort. When the occupation was not available at the age of 35 , exactly, we extracted those participants' occupational information from the next ascending year. Furthermore, if there was no existing occupation in the ascending direction, we extracted the occupation from the nearest descending year. Occupational information in STATIV entailed a variable consisting of the SSYK96 coding system (see Statistics Sweden [SCB], 1998). SSYK96 is a Swedish adaptation of the International Standard Classification of Occupations (ISCO-88; International Labour Office in Geneva, 1990) and the closely related ISCO-88 (COM), which is used when reporting and compiling statistics within the European Union (EU). It divides occupations into hierarchical frameworks based on two key concepts, namely the kind of work performed and skill level (SCB, 1998). Kind of work pertains to tasks or duties to be executed and is grouped into 10 major occupational fields, whereas skill level is evaluated based on the qualification and specialization requirements of a given occupation. We used the second-most detailed three-digit scale of the Swedish Standard Classification of Occupations (SSYK-3), containing 113 occupations.

For calculating the cognitive demands of chosen occupation, we used a coding scheme developed by Dekhtyar et al. (2018). Dekhtyar et al. (2018) conducted an extensive evaluation of each occupation in the SSYK-paradigm using nine independent coders who were prompted to rate, in two separate questions, how common both numerical and verbal tasks were in a given 
occupation on a scale ranging from 1 (not at all common) to 7 (numerical/verbal tasks are routinely demanded). Following this procedure, the authors created a cognitive demands score by subtracting the verbal from the numerical demands score. A negative cognitive demands score indicates that a given occupation is more numerically demanding, whereas a positive score indicates that it is more verbally demanding. For our studies, we deployed this cognitive demands score with one variation; as the coding scheme was developed on the four-digit SSYK-4 scale, which yields more refined specializations within an occupational domain, we averaged the cognitive demands scores onto our SSYK-3 level keys. Therefore, although this will not provide identical or such refined scores as in Dekhtyar et al. $(2018)^{1}$, we can untangle the cognitive demands across our 113 occupations with sufficient precision. The distribution in the occupational demands score ranged from -3.94 to 4.50 in both studies, with 'Mathematicians and Statisticians' scored as the most numerically demanding occupation, and 'Pastors' scored as the most verbally demanding occupation.

Covariates. In this study, five control variables served as covariates in model computation: Grade Point Average (GPA), mother's age at birth, mother's education level, sibship type (identical parents vs. not identical parents), ethnicity (Swedish vs. other), and age difference (in years) between the siblings.

Analytical approach. All analyses were performed in the $\mathrm{R}$ environment ( $\mathrm{R}$ Core Team, 2016). Multiple linear regression models were computed to assess the relationship between sibship composition and our two target outcomes: (1) Academic strengths at age 16, and (2) Cognitive demands of the chosen occupation at age 35 . For each target outcome, we first conducted a multiple regression model with sex (boys vs. girls) as the predictor for establishing the expected impact of sex differences. This was followed by two separate models, the first including the two sibship compositions among girls (Study 1: GGG vs. BGB; Study 2: GG vs. BG) and the second including the two sibship compositions among boys (Study 1: BBB vs. GBG, Study 2: BB vs. GB). In each model, we controlled for the following covariates: GPA, mother's age at birth, mother's education level, sibship type (identical parents vs. not identical parents), ethnicity (Swedish vs. other), and age difference (in years) between the siblings (Study 1: age difference between the first-born and mid-born child, and age difference between the last-born and mid-born child; Study 2: age difference between the first-born and last-born child). Together with the regression coefficients, we report Cohen's $f^{2}$ effect sizes using the sjstats package (Lüdecke, 2018). The $f^{2}$ effect sizes are interpreted following Cohen's (1988) guidelines, where $f^{2} \geq 0.02$ is a small effect, $f^{2} \geq 0.15$ is a moderate effect, and $f^{2} \geq 0.35$ is a large effect.

Employing a $2 \times 3$ factorial ANOVA, we also examined whether the association between cognitive demands of chosen occupation at age 35 and sibship composition was moderated by academic strengths at age 16. Occupational demands served as the dependent variable, whereas the main effect of sibship composition and academic strengths, and their interaction term, served as predictors of interest. The academic strengths score was categorized into three levels in these analyses: those participants with a score above 0 were defined as having "verbally aligned academic strengths", those with a score of 0 points were defined as having "equally weighted academic strengths", and those having a score below 0 were defined as having a "numerically aligned academic strengths". As before, the model results were adjusted for our pre-defined covariates. If a significant sibship composition $\times$ academic strengths interaction was observed, we followed up this effect with tests of the simple main effects on each of the three levels of the academic strengths factor.

\section{Results}

Descriptives. In Supplemantary Materials, we provide descriptive statistics of the academic strengths score, occupational demands score, school subjects scores, and the variables fed into the proposed regression models (including their intercorrelations) in Study 1 (Supplementary Materials S1; Fig. 1, Tables 1, 2, Fig. 2) and Study 2 (Supplementary Materials S2; Fig. 1, Tables 1, 2, Fig. 2). Demographic characteristics of the four groups in Study 1 and Study 2 can be found in Table 1 . Overall, the birth cohorts were relatively equally spread in our study samples, both across gender and group. The only exception was the 1982 cohort which was very few in number, stemming from the fact that these participants had started school a year ahead of their actual age cohort. However, as we sought to maximize the number of children that had school grades from 9th grade between 1988 and 1997, we decided to include those who graduated one year early as well. Of the mid-born children in Study $1,90.2 \%$ had siblings who had the same father and mother, and $41.7 \%$ had a Master's degree. The most representative occupation at age 35 was Professional occupations (29.0\%), followed by Technicians and associate professionals (22.5\%) and Service workers and sales personnel (16.7\%). In Study 2, the vast majority of the last-born children (94.6\%) had an older sibling with identical parents. At age 35, more than half of the participants (55.7\%) had a Master's degree. The most represented occupation in Study 2 was Professional occupations (30.8\%), followed by Technicians and associate professionals (23.9\%).

\section{Multiple regression analyses}

Study 1. Table 2 depicts an aggregated table of our four target outcomes grouped by sex and sibship composition. The full output (including the coefficients from the covariates) from a given model can be found in Supplementary Materials (Supplementary Materials S1; Tables 3-8).

Academic strengths at age 16. We observed a statistically significant relationship between academic strengths and sex after controlling for the covariates $(\beta=-0.584,95 \%$ CI $[-0.614$, $\left.-0.555], p<0.001, f^{2}=0.325\right)$. This indicates that the academic strengths score among girls was more verbally aligned, whereas the academic strengths of the boys were more numerically aligned, which is in line with previous findings (Dekhtyar et al., 2018). The within-sex analyses showed that sibship composition was not significantly associated with academic strengths among either girls $(\beta=0.008,95 \%$ CI $[-0.035,0.051], p=0.715$, $\left.f^{2}=0.003\right)$ or boys $(\beta=-0.012,95 \%$ CI $[-0.054,0.030]$, $\left.p=0.578, f^{2}=0.005\right)$, suggesting that academic strengths remain unaffected, regardless of whether a girl or boy grows up with same- or opposite-sex siblings (see Fig. 1A).

Cognitive demands of chosen occupation at age 35 . We found a statistically significant association between sex and cognitive demands of chosen occupation at age $35(\beta=-0.813,95 \% \mathrm{CI}$ $\left.[-0.859,0.803], p<0.001, f^{2}=0.194\right)$. This indicates that girls, relative to boys, tend to choose occupations that are more verbally demanding, whereas boys tend to choose occupations that are more numerically demanding. In the within-sex analyses, we did not observe a significant association between cognitive demands of chosen occupation and sibship composition among girls $\left(\beta=0.013,95 \%\right.$ CI $\left.[-0.029,0.055], p=0.553, f^{2}=0.010\right)$. However, among boys, we did observe a trend towards statistical significance $(\beta=-0.040,95 \%$ CI $[-0.082,0.003], p=0.065$, $\left.f^{2}=0.017\right)$. The results from the latter analysis provide tentative 


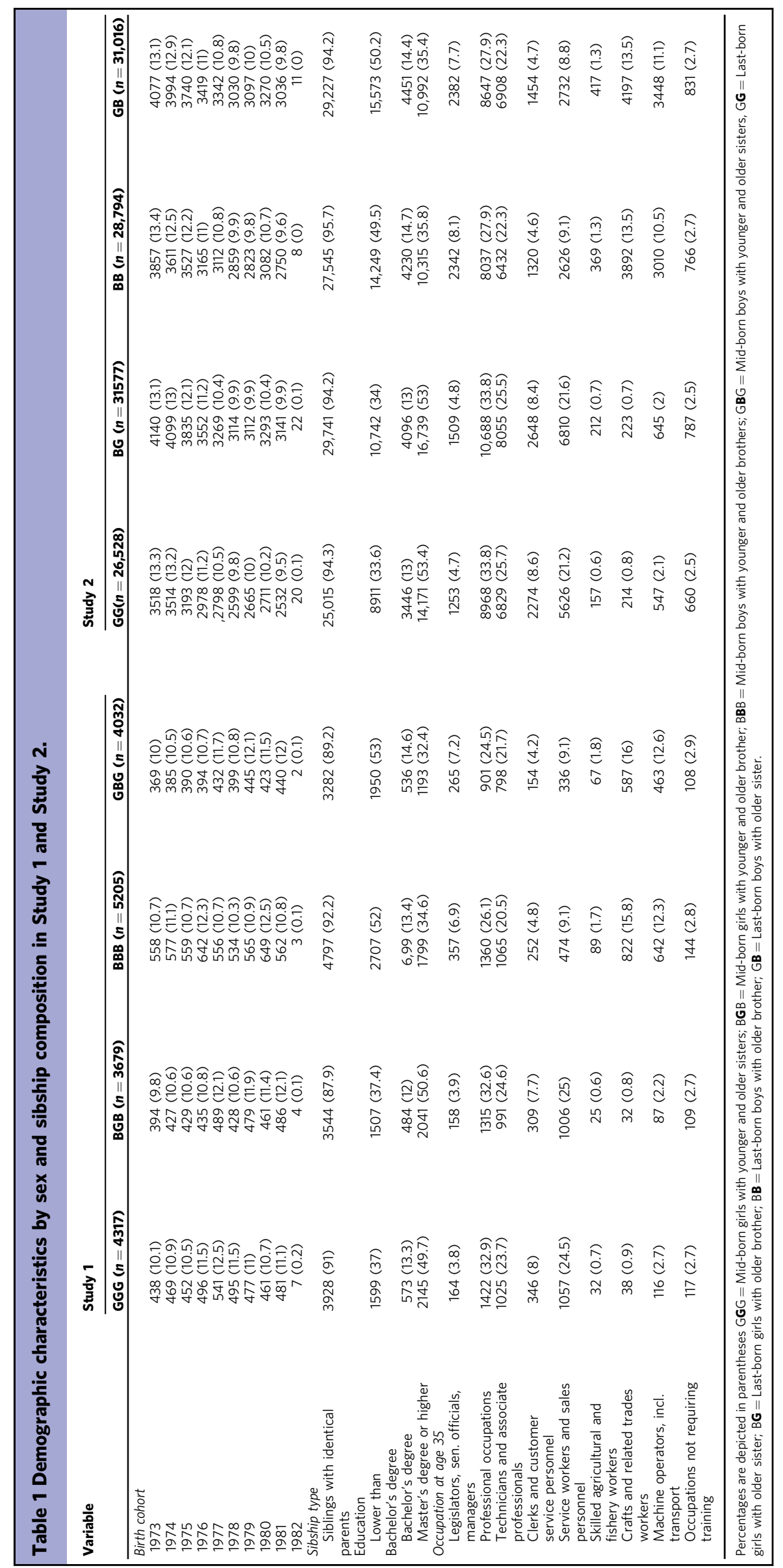


Table 2 Regression table summarizing the differences in academic strengths and cognitive demands of chosen occupation grouped by sex (across sexes) and sibship composition (within sexes) in Study 1.

\begin{tabular}{|c|c|c|c|c|c|}
\hline Comparison & B & B $95 \%$ Cl [LL, UL] & $\beta$ & Sig. & Cohen's $f^{2}$ \\
\hline Girls vs. Boys & -0.800 & {$[-0.839,-0.76]$} & -0.584 & $<0.001$ & 0.325 \\
\hline GGG vs. $B \mathbf{G B}$ & 0.010 & {$[-0.044,0.064]$} & 0.008 & 0.715 & 0.003 \\
\hline \multicolumn{6}{|c|}{ Cognitive demands of chosen occupation at age 35} \\
\hline Girls vs. Boys & -1.673 & {$[-1.730,-1.616]$} & -0.831 & $<0.001$ & 0.426 \\
\hline GGG vs. $B \mathbf{G B}$ & 0.024 & {$[-0.055,0.103]$} & 0.013 & 0.553 & 0.010 \\
\hline
\end{tabular}

Note: The group to the left served as the intercept in the model. In all models, the effect of sex/sibship composition was adjusted for GPA, mother's age at birth, mother's education level, sibship type, ethnicity, and age differences between the siblings. Bolding in the "Sig."-column indicates statistical significance at $\alpha<0.05)$. LL and UL indicate the lower and upper limits of the confidence interval. All coefficients, including the covariates from each model, can be seen in Supplementary Materials S1.

support for the fact that boys with a younger and older sister tend to choose more numerically demanding occupations as compared to boys with a younger and older brother (see Fig. 1B).

Study 2. Table 3 depicts an aggregated table of our two target outcomes grouped by sex and sibship composition. The full output (including the coefficients from the covariates) from a given model can be found in Supplementary Material (Supplementary Materials S2; Tables 3-8).

Academic strengths at age 16. We observed a statistically significant relationship between academic strengths and sex, after adjusting for our control variables $(\beta=-0.574,95 \%$ CI $[-0.585$, $\left.-0.563], p<0.001, f^{2}=0.320\right)$. This indicates that academic strengths among girls were more verbally aligned, whereas the academic strengths of the boys were more numerically aligned. The within-sex analyses showed that sibship composition was not significantly associated with academic strengths among either girls $\left(\beta=0.009,95 \% \mathrm{CI}[-0.007,0.025], p=0.262, f^{2}=0.003\right)$ or boys $\left(\beta=-0.004,95 \%\right.$ CI $\left.[-0.020,0.012], p=0.658, f^{2}=0.001\right)$, suggesting that academic strengths remain unaffected regardless of whether an individual, girl or boy, grows up with a same-sex or opposite-sex sibling (see Fig. 1C).

Cognitive demands of chosen occupation at age 35 . We observed a statistically significant association between sex and cognitive demands of chosen occupation $(\beta=-0.810,95 \%$ CI $[-0.821$, $\left.-0.799], p<0.001, f^{2}=0.411\right)$. This indicates that girls, relative to boys, tend to choose occupations that are more verbally demanding, whereas boys tend to choose occupations that are more numerically demanding. In the within-sex analyses, we did not observe a significant association between sibship composition among girls and cognitive demands of chosen occupation $\left(\beta=-0.008,95 \%\right.$ CI $\left[-0.024,0.008, p=0.304, f^{2}=0.001\right)$. However, among the boys, we did observe a significant association between sibship composition and cognitive demands of chosen occupation $(\beta=-0.019$, 95\% CI $[-0.035,-0.003], p=0.022$, $f^{2}=0.007$ ). In line with the direction observed in Study 1 , this indicates that last-born boys with an older brother tend to choose more verbally demanding occupations as compared to last-born boys with an older sister (for a visual illustration, see Fig. 1D).

Moderator analyses. Next, we examined whether the association between cognitive demands of chosen occupation at age 35 and sibship composition was moderated by the participants' academic strengths at age 16 using a 2 (sibship composition) $\times 3$ (academic strengths) factorial ANOVA.
Study 1. Among girls in Study 1 (i.e., GGG vs. BGB), the results showed a main effect of academic strengths, $F(1,8336)=14.779$, $p<0.001, f^{2}=0.06$, indicating that verbally aligned girls at age 16 were more likely to choose a verbally demanding occupation at 35 years old (see also Fig. 2A). The main effect of sibship composition was not statistically significant, $F(1,8336)=0.346$, $p=0.556, f^{2}=0.006$. Likewise, the sibship composition $\times$ academic strengths interaction was not statistically significant, $F(2,8336)=1.278, p=0.279, f^{2}=0.018$.

Among the boys (i.e., BBB vs. GBG), we observed a significant main effect of academic strengths on cognitive demands of chosen occupation, $F(1,8871)=61.537, p<0.001, f^{2}=0.118$, indicating that numerically aligned boys at age 16 were more likely to choose a numerically demanding occupation at 35 years old (see also Fig. 2A). However, neither the main effect of sibship composition, $F(1,8871)=2.917, p=0.088, f^{2}=0.018$, nor the interaction term between sibship composition and academic strengths, $F(2,8871)=2.451, p=0.086, f^{2}=0.024$ was statistically significant.

Study 2. Starting with the girls in Study 2 (i.e., GG vs. BG), the results revealed a main effect of academic strengths on occupational choice, $F(1,58,093)=79.418, p<0.001, f^{2}=0.052$. This indicates that girls whose academic strengths were verbally aligned at age 16 were more likely to work in more verbally demanding occupations at age 35 (see also Fig. 2B). However, we neither observed a main effect of sibship composition on occupational choices $F(1,58,093)=1.081, p=0.299, f^{2}=0.004$ nor a sibship composition $\times$ academic strengths interaction on occupational choice, $F(2,58,093)=1.371, p=0.254, f^{2}=0.007$.

With respect to boys (i.e., BB vs. GB), we also observed a significant main effect of academic strengths on cognitive demands of chosen occupation, $F(1,59,798)=386.138$, $p<0.001, f^{2}=0.114$, suggesting that those boys with more numerically aligned cognitive abilities at age 16 were more likely to work in numerically demanding occupations. We also observed a statistically significant main effect of group on occupational demands, $F(1,59,798)=5.117, p=0.024, f^{2}=0.009$, indicating that last-born boys with an older sister were more likely to choose a numerically demanding occupation at age 35 relative to lastborn boys with an older brother. Importantly, we observed a statistically significant sibship composition $\times$ academic strengths interaction, $F(2,59,798)=3.527, p=0.029, f^{2}=0.011$. Follow-up analyses on this effect showed that a boy with verbally aligned academic strengths at age 16 was less likely to choose a numerically demanding occupation at age 35 if his older sibling was a brother, $F(1,15,274)=7.826, p=0.005, f^{2}=0.023$ (see also Fig. 2B). Those boys that demonstrated equally weighted academic strengths at age 16 did not differ from each other on 
(A) Study 1: Academic strengths at age 16

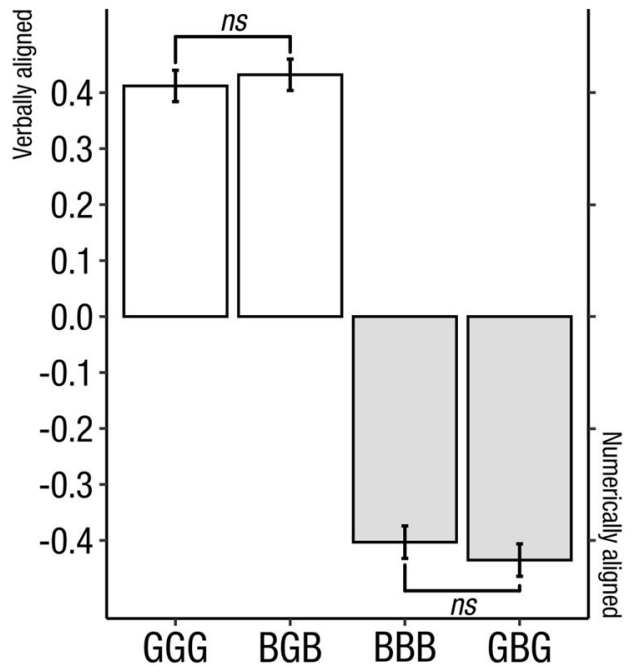

(C) Study 2: Academic strengths at age 16

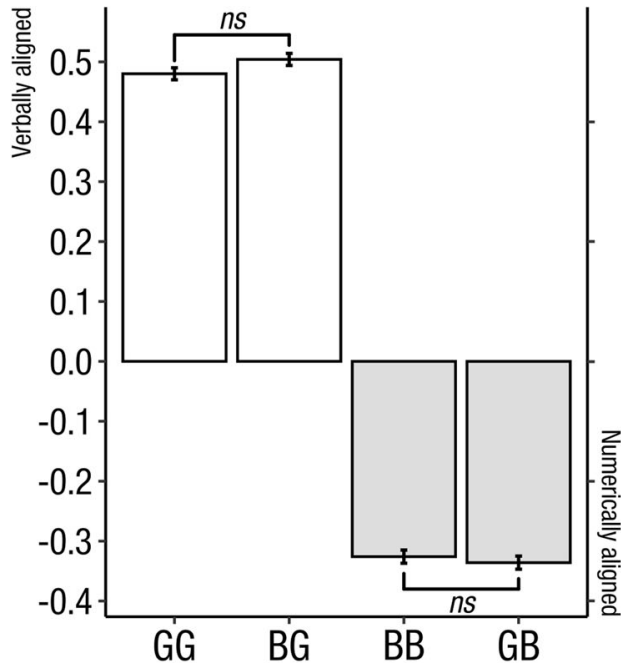

(B) Study 1: Cognitive demands

of chosen occupation at age 35

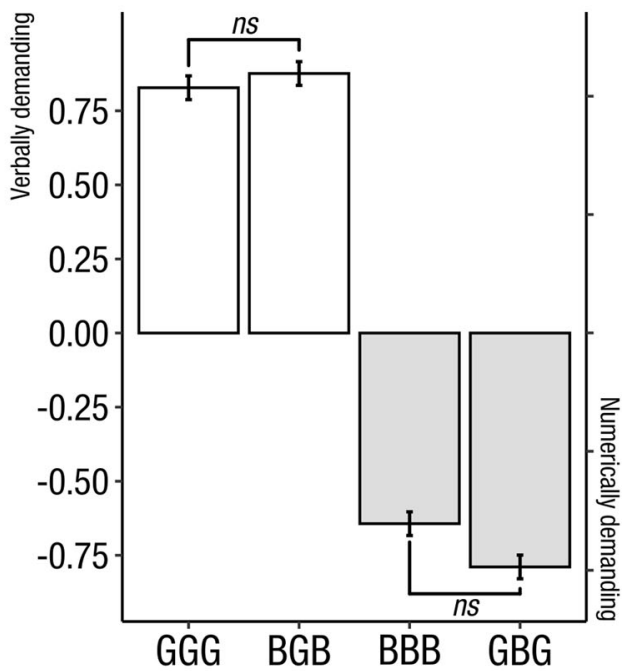

(D) Study 2: Cognitive demands of chosen occupation at age 35

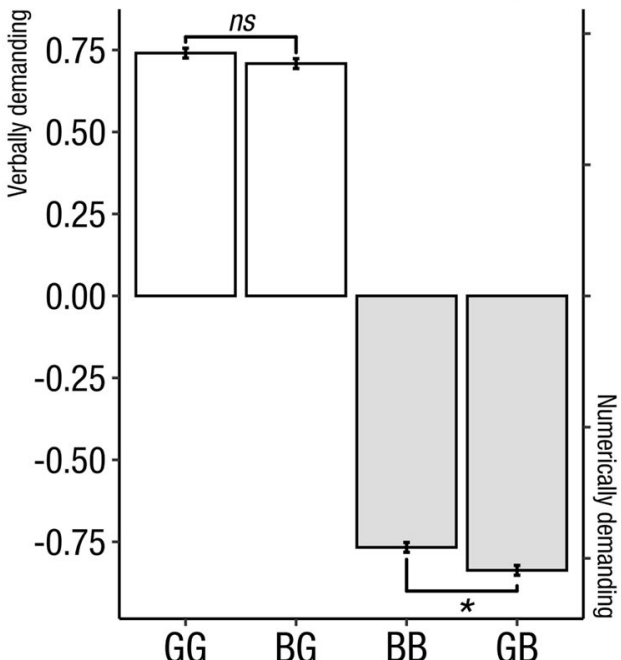

Fig. 1 Illustrations of the academic strengths score and cognitive demands of chosen occupation grouped by sibship composition and sex. Academic strengths at age 16 grouped by sibship composition in $\mathbf{A}$ Study 1 and $\mathbf{C}$ Study 2. Positive values indicate higher grades in verbal/language subjects, whereas negative values indicate higher grades in numerical/technical subjects. Cognitive demands of chosen occupation at age 35 grouped by sibship composition in B Study 1, and D Study 2. Positive values indicate higher verbal demands in occupation, whereas negative values indicate higher numerical demands in occupation. GGG = Mid-born girls with younger and older sisters; $B G B=$ Mid-born girls with younger and older brothers; $B B B=$ Mid-born boys with younger and older brothers; GBG = Mid-born boys with younger and older sisters. Note: All means are adjusted for GPA, mother $\backslash s$ age at birth, mother $\backslash s$ education level, sibship type, ethnicity (Swedish or other), and age differences between the siblings. Whiskers represent the standard error of the mean. ns $=$ not significant; ${ }^{*} p<0.05$.

occupational choices, regardless of the sex of their older sibling, $F(1,17914)=0.363, p=0.547, f^{2}=0.005$. Likewise, those boys with numerically aligned academic strengths at age 16 did not differ from each other in occupational choices, $F(1$, $26,598)=3.263, p=0.071, f^{2}=0.011$, regardless of the sex of their older sibling.

Sensitivity analyses. In order to assure that the investigated siblings were raised together, we performed sensitivity analyses wherein we included only those siblings who had identical parents. Although this approach does not entirely rule out the possibility that siblings have been raised in different families, it certainly reduces this risk. Moreover, we probed for possible spacing effects by specifying an interaction term between sibship composition and age difference (in years) between the first-born and last-born child on the respective target outcome. The results from the sensitivity analyses concurred strongly with those results obtained from the main analyses, suggesting that results were unaffected by sibship type and spacing effects (see Supplementary Materials S3 for more details regarding the sensitivity analyses).

\section{Discussion}

We investigated whether our tendency to be more verbally or numerically aligned academically and in occupational choices is influenced by growing up in a more or less gender-typed environment, which we operationalized as same- or opposite-sex 
Table 3 Regression table summarizing the differences in academic strengths and cognitive demands of chosen occupation grouped by sex (across sexes) and sibship composition (within sexes) in Study 2.

\begin{tabular}{|c|c|c|c|c|c|}
\hline Comparison & B & B $95 \% \mathrm{Cl}[\mathrm{LL}, \mathrm{UL}]$ & $\beta$ & Sig. & Cohen's $f^{2}$ \\
\hline Girls vs. Boys & -0.779 & {$[-0.794,-0.764]$} & -0.574 & $<0.001$ & 0.320 \\
\hline GG vs. BG & 0.012 & {$[-0.009,0.032]$} & 0.009 & 0.262 & 0.003 \\
\hline \multicolumn{6}{|c|}{ Cognitive demands of chosen occupation at age 35} \\
\hline Girls vs. Boys & -1.644 & {$[-1.666,-1.622]$} & -0.810 & $<0.001$ & 0.411 \\
\hline GG vs. BG & -0.016 & {$[-0.046,0.014]$} & -0.008 & 0.304 & 0.001 \\
\hline
\end{tabular}

The group to the left served as the intercept in the model. In all models, the effect of sex/sibship composition was adjusted for GPA, mother's age at birth, mother's education level, sibship type, ethnicity, and age differences between the siblings. Bolding in the "Sig."-column indicates statistical significance at $\alpha<0.05)$. LL and UL indicate the lower and upper limits of the confidence interval. All coefficients, including the covariates from each model, can be seen in Supplementary Materials S2.
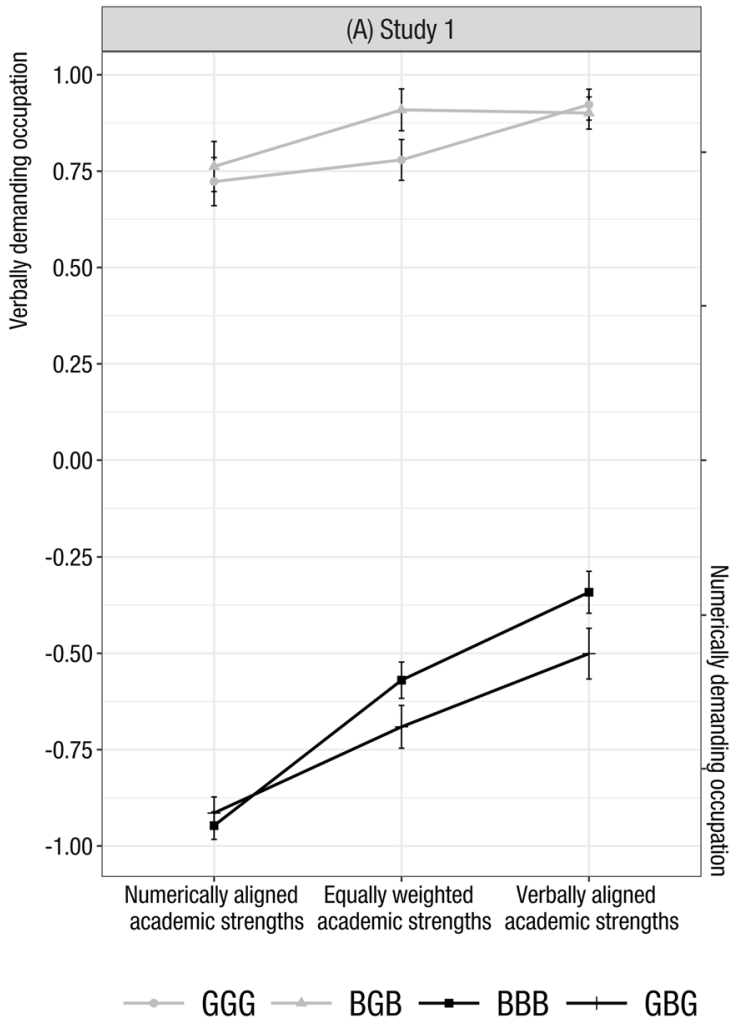

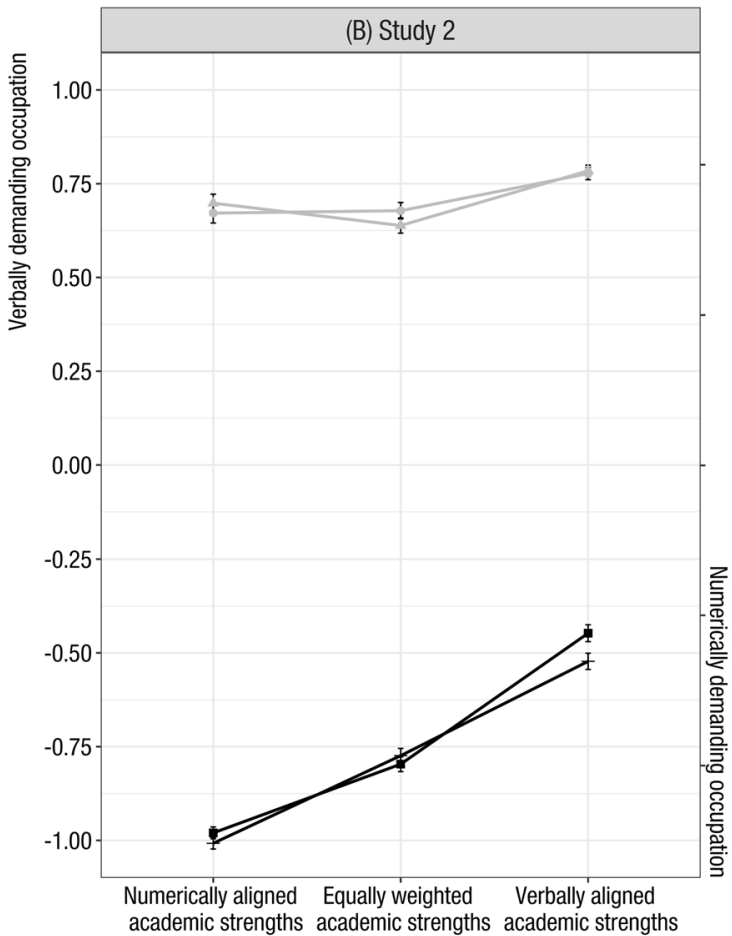

$\mathrm{GG} \longrightarrow \mathrm{BG} \longrightarrow \mathrm{BB} \longrightarrow \mathrm{GB}$

Fig. 2 Cognitive demands of chosen occupation at age 35 ( $y$-axis) grouped by academic strengths at age 16 ( $x$-axis) and by sibship composition among girls (coloured in grey) and boys (coloured in black) in A Study 1, and B Study 2. GGG = mid-born girls with younger and older sisters; BGB = mid-born girls with younger and older brother; $\mathrm{BBB}=$ mid-born boys with younger and older brothers; $\mathrm{GBG}=$ mid-born boys with younger and older sisters. $\mathrm{GG}=$ last-born girls with older sister; $\mathrm{BG}=$ last-born girls with older brother; $\mathrm{BB}=$ last-born boys with older brother; $\mathrm{GB}=$ last-born boys with older sister. Whiskers represent standard error of the mean.

sibling composition. Utilizing high-quality register data from Sweden on full cohorts, Study 1 focused on the mid-born child in 3 -sibling families with either same- or opposite-sex siblings, whereas Study 2 focused on the last-born child in 2-sibling families with either a same- or opposite-sex older sibling. In both studies, we examined the impact of sibship composition on individuals' academic strengths at age 16 and on the cognitive demands of chosen occupations at age 35. Importantly, we found no evidence supporting the notion that the degree to which one's academic strengths is verbally or numerically aligned at age 16 is affected by growing up in a more or less female or male environment, in spite of these academic strengths showing substantial sex differences, with boys being more numerically aligned and girls being more verbally aligned. However, whereas sibling composition did not affect females' chosen occupations at age 35 , it did so for males. At age 35, males raised with opposite-sex siblings had chosen occupations more numerically aligned than males with same-sex siblings.

As expected, the sex differences in academic strengths at age 16 in both Study 1 and 2 showed that girls tend to be more verbally aligned, whereas boys tend to be more numerically aligned (Dekhtyar et al., 2018; Stoet and Geary, 2018; Strandqvist et al., 2019). Importantly, however, we did not observe an effect of sibship composition on academic strengths. Instead, girls with same-sex siblings were equally verbally aligned in their academic strengths as girls with opposite-sex siblings, and the same was true for boys, for whom numerical alignment persisted in the face of sibship composition. These null effects held true for the 
sensitivity analyses wherein we included only those children that shared identical parents with his/her sibling(s), under the assumption that these siblings spent more time together than those not sharing identical parents. Our sensitivity analyses also showed that spacing effects among siblings were non-significant, that is, whether siblings are more or less close in age did not affect the outcome (Barclay and Kolk, 2017; Grätz, 2018). Thus, being exposed to an either female or male environment in the form of same- or opposite-sex siblings does not exert enough influence to alter how verbally or numerically aligned one's academic strength is at 16 years of age.

As expected (Dekhtyar et al., 2018), we observed sex differences in cognitive demands of chosen occupation at age 35, indicating that girls select occupations that are more verbally than numerically demanding and boys tend to choose occupations that are more numerically than verbally demanding. We also found that mid-born boys in 3-sibling families and last-born boys in 2-sibling families tended to choose occupations with more numerical demands if their oldest/older sibling was a girl, although it should be noted that effect sizes were very small (Study 1 Cohen's $d=0.034$; Study 2 Cohen's $d=0.014)^{2}$ and that the relationship only approached significance in Study 1. The same tendency was found in the subsequent moderator analysis, showing that boys with verbally aligned academic strengths at age 16 were less likely to choose a numerically demanding occupation at age 35 if their older sibling was a brother. Reasons for why boys with an older sister tend to choose more numerically demanding occupations are likely manifold, but one explanation could be that one's "awareness" of gender-typicality is attenuated when interacting with opposite-sex siblings. Specifically, a boy growing up with opposite-sex siblings might start behaving more gender-stereotypically in order to differentiate himself from the sister(s) (Brenøe, 2018), potentially reinforced by increased gender-differentiating parenting that typically accompanies opposite-sex sibship composition (Feinberg et al., 2003). An explanation for why a relationship between sibship composition and occupational choices was found among boys, but not among girls, remains an open question.

A finding worth noting was that we found no relationship between sibship composition and academic strengths, whereas we did so for cognitive demands of chosen occupation among boys, suggesting that one's academic strengths are more resilient to environmental factors than one's choice of occupation. This is in line with earlier findings demonstrating that cognitive performance is stable from childhood to old age (Deary et al., 2004) and largely heritable (Plomin and Deary, 2015). Occupational choices, on the other hand, may be more vulnerable to the influence of societal conditions and alterations, and, thereby, also vulnerable to environmental influences of same- or opposite-sex siblings, a relationship which has been demonstrated in earlier studies (Brenøe, 2018; Gabay-Egozi et al., 2020).

A potential limitation of the study is our basis for an academic strengths score on school grades rather than on cognitive test performance, considering that non-cognitive factors and teacher bias may influence grade assignment (Beilock et al., 2010). However, the grades used were also based on the students' standardized national test performance in Swedish and Math. Furthermore, research has demonstrated that cognitive performance can be reliably estimated using school grades (Roth et al., 2015), strengthening the notion that we capture individuals' relative strengths in verbal and numerical abilities. Another potential confound related to occupational demands is whether males and females in this study worked in occupations of comparable skill level, despite working in occupations of different verbal-numerical demands. As our main analyses were concentrated mostly on within-sex comparisons where the only manipulation was denoted to different sibship compositions, we are rather confident that such across-sex effects, if existing here, would not affect the results to any greater extent.

In summary, having a brother(s) or sister(s) of the same or opposite sex does not affect one's tendency to be more or less verbally or numerically aligned-our academic strengths, which show reliable sex differences, appear resistant to growing up in a more or less gender-typed environment. In contrast, boys with an older sister tend to choose more numerically demanding occupations relative to boys with an older brother. However, the effect of this relationship was very small, and not observed among girls, indicating that most of the occupational paths are explained by factors unrelated to sibship composition.

Ethics statement. The study was approved by the Swedish Ethical Review Authority (no. 2019-06241). In accordance with the Swedish Ethical Review Authority, informed consent is not required from individuals included in the Swedish registries. All methods used to analyze the registry data were carried out in accordance with relevant guidelines and regulations.

\section{Data availability}

This study was produced under the Swedish Statistics Act, according to which privacy concerns restrict the availability of register data for research. Aggregated data can be made available by the authors, subject to ethical vetting. Enquiries should be made to the corresponding author.

Received: 20 March 2021; Accepted: 31 August 2021; Published online: 29 September 2021

\section{Notes}

1 One such example is the occupation of translators, which was rated with a score of 4 in the original coding system. However, with the less refined SSYK-3 level, we were not able to untangle translators as a distinct and separated occupation. Instead, translators were categorized under a broader category, labelled as social and linguistic sciences, and whose score was 1.25 on the verbal-numerical scale in the present study.

2 For the sake of interpretability in the discussion, we converted the Cohen's $f^{2}$ values to Cohen's $d$ values (formula: $d^{2}=f^{2} \times 2$ ).

\section{References}

Asperholm M, Högman N, Rafi J, Herlitz A (2019) What did you do yesterday? A meta-analysis of sex differences in episodic memory. Psychol Bull 145(8):785-821

Barclay KJ, Kolk M (2017) The long-term cognitive and socioeconomic consequences of birth intervals: a within-family sibling comparison using Swedish register data. Demography 54:459-84

Beilock SL, Gunderson EA, Ramirez G, Levine SC (2010) Female teachers' math anxiety affects girls' math achievement. Proc Natl Acad Sci USA 107(5):1860-63

Brenøe A (2018) Origins of gender norms: sibling gender composition and women's choice of occupation and partner. IZA Discussion Paper No. 11692. http://ftp.iza.org/dp11692.pdf

Brim OG (1958) Family structure and sex role learning by children: a further analysis of Helen Koch's data. Sociometry 21(1):1-16

Ceci SJ, Ginther DK, Kahn S, Williams WM (2014) Women in academic science: a changing landscape. Psychol Sci Public Interest 15(3):75-141

Cohen J (1988) Statistical power analysis for the behavioral sciences. Lawrence Erlbaum Associates, Hillsdale

Deary IJ, Whiteman MC, Starr JM, Whalley LJ, Fox HC (2004) The impact of childhood intelligence on later life: following up the scottish mental surveys of 1932 and 1947. J Pers Soc Psychol 86(1):130-147

Dekhtyar S, Weber D, Helgertz J, Herlitz A (2018) Sex differences in academic strengths contribute to gender segregation in education and occupation: a longitudinal examination of 167,776 individuals. Intelligence 67:84-92

Detlefsen L, Friedl A, Lima de Miranda K, Schmidt U, Sutter M (2018). Are economic preferences shaped by the family context? The impact of birth order and siblings' sex composition on economic preferences. IZA Discussion Paper No. 11949. http://ftp.iza.org/dp11949.pdf 
Feinberg ME, McHale SM, Crouter AC, Cumsille P (2003) Sibling differentiation: sibling and parent relationship trajectories in adolescence. Child Dev 74(5):1261-1274

Gabay-Egozi L, Grieger L, Nitsche N (2020). In my brother's footstep or shadow? Siblings' compositional characteristics and fender differences in STEM major. Max Planck Institute Demographic Research https://www.demogr.mpg.de/ papers/working/wp-2020-031.pdf

Golsteyn BHH, Magnée CAJ (2020) Does sibling gender affect personality traits? Econ Educ Rev 77(2020):1020162

Grätz M (2018) Competition in the family: inequality between siblings and the intergenerational transmission of educational advantage. Sociol Sci 5:246-69

Grotevant HD (1978) Sibling constellations and sex typing of interests in adolescence. Child Dev 49:540-42

Hamilton WD (1967) Extraordinary sex ratios. Science 156:477-88

Hyde JS, Linn MC (1988) Gender differences in verbal ability: a meta-analysis. Psychol Bull 104(1):53-69

Koch HL (1955) Some personality correlates of sex, sibling position, and sex of sibling among five-and six-year-old children. Genet Psychol Monogr 52:3-50

Lindberg SM, Hyde JS, Petersen JL, Linn MC (2010) New trends in gender and mathematics performance: a meta-analysis. Psychol Bull 136(6):1123-35

Lüdecke D (2018). sjstats: statistical functions for regression models. R Package version 0.14 .1

Maylor EA, Reimers S, Choi J, Collaer ML, Peters M, Silverman I (2007) Gender and sexual orientation differences in cognition across adulthood: age is kinder to women than to men regardless of sexual orientation. Arch Sex Behav 36:235-49

McHale SM, Crouter AC, Tucker CJ (1999) Family context and gender role socialization in middle childhood: comparing girls to boys and sisters to brothers. Child Dev 70(4):990-1004

Peter N, Lundborg P, Mikkelsen S, Webbink D (2018) The effect of a sibling's gender on earnings and family formation. Labour Econ 54:61-78

Plomin R, Deary I (2015) Genetics and intelligence differences: five special findings. Mol Psychiatry 2015(20):98-108

R Core Team (2016). R: A language and environment for statistical computing. $R$ Foundation for Statistical Computing, Vienna, Austria.

Roth B, Becker N, Romeyke S, Schäfer S, Domnick F, Spinath FM (2015) Intelligence and school grades: a meta-analysis. Intelligence 53:118-37

Statistics Sweden (2018) Documentation of STATIV. Statistics Sweden, Stockholm

Statistics Sweden (2020) Women and men in Sweden 2020: facts and figures. Statistiska Centralbyrån, Stockholm

Statistics Sweden (1998). SSYK 96: Standard för svensk yrkesklassificering 1996 [Swedish Standard Classification of Occupations 1996]. Official Statics of Sweden

Stoet G, Geary DC (2013) Sex differences in mathematics and reading achievement are inversely related: within- and across-nation assessment of 10 years of PISA data. PLoS ONE 8(3):e57988

Stoet G, Geary DC (2018) The gender-equality paradox in science, technology, engineering, and mathematics education. Psychol Sci 29(4):581-93

Strandqvist A, Örtqvist L, Frisén L, Nordenskjöld A, Herlitz A, Nordenström A (2019) No difference in cognitive performance or gender role behavior between men with and without hypospadias. Horm Behav 109:64-70

Su R, Rounds J, Armstrong PI (2009) Men and things, women and people: a metaanalysis of sex differences in interests. Psychol Bull 135(6):859-84
Todd BK, Fischer RA, Di Costa S, Roestorf A, Harbour K et al. (2018) Sex differences in children's toy preferences: a systematic review, meta-regression, and meta-analysis. Infant Child Dev 27(2):e2064

Voyer D, Voyer SD (2014) Gender differences in scholastic achievement: a metaanalysis. Psychol Bull 140(4):1174-1204

Voyer D, Voyer SD, Bryden MP (1995) Magnitude of sex differences in spatial abilities: a meta-analysis and consideration of critical variables. Psychol Bull 117(2):250-70

Voyer D, Voyer SD, Saint-Aubin J (2017) Sex differences in visual-spatial working memory: a meta-analysis. Psychon Bull Rev 24:307-34

Weber D, Skirbekk V, Freund I, Herlitz A (2014) The changing face of cognitive gender differences in Europe. Proc Natl Acad Sci USA 111(32):11673-78

\section{Acknowledgements}

A.H. received funding from the Swedish Reseach Council (Grant No. 2018-01334). R.B. received funding from the Swedish Reseach Council (Grant No. 2018-01876).

\section{Funding}

Open access funding provided by Karolinska Institute.

\section{Competing interests}

The authors declare no competing interests.

\section{Additional information}

Supplementary information The online version contains supplementary material available at https://doi.org/10.1057/s41599-021-00897-5.

Correspondence and requests for materials should be addressed to Daniel Fellman or Agneta Herlitz.

Reprints and permission information is available at http://www.nature.com/reprints

Publisher's note Springer Nature remains neutral with regard to jurisdictional claims in published maps and institutional affiliations.

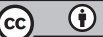

Open Access This article is licensed under a Creative Commons Attribution 4.0 International License, which permits use, sharing, adaptation, distribution and reproduction in any medium or format, as long as you give appropriate credit to the original author(s) and the source, provide a link to the Creative Commons license, and indicate if changes were made. The images or other third party material in this article are included in the article's Creative Commons license, unless indicated otherwise in a credit line to the material. If material is not included in the article's Creative Commons license and your intended use is not permitted by statutory regulation or exceeds the permitted use, you will need to obtain permission directly from the copyright holder. To view a copy of this license, visit http://creativecommons.org/ licenses/by/4.0/.

(C) The Author(s) 2021 